Pacific Journal of Mathematics

THE CALCULATION OF AN INVARIANT FOR TO 


\title{
THE CALCULATION OF AN INVARIANT FOR TOR
}

\author{
BRIAN D. WICK
}

Let $\lambda$ be a limit ordinal such that $\lambda$ is not cofinal with $\omega$ and let $G=\operatorname{Tor}(A, B)$ where $A$ and $B$ are reduced $p$-groups. It is shown that the invariant defined to be the dimension of the $Z / p Z$-vector space $p^{\lambda} \operatorname{Ext}\left(Z\left(p^{\infty}\right), G / p^{\lambda} G\right) / p^{\lambda+1} \operatorname{Ext}\left(Z\left(p^{\infty}\right), G / p^{\lambda} G\right)$ is zero. If $A, B$ and $\operatorname{Tor}(A, B)$ are three totally projective $p$-groups then either $A$ or $B$ must be the direct sum of countable $p$-groups.

1. Introduction. Warfield introduced in [6] the class of $S$-groups and showed that these groups can be distinguished by a collection of invariants. These invariants for the group $G$ consisted of the classical Ulm invariants and the invariant $k\left(p^{\lambda}, G\right)$, defined to be the dimension of the $Z / p Z$-vector space $p^{\lambda} \operatorname{Ext}\left(Z\left(p^{\infty}\right), G / p^{\lambda} G\right) / p^{\lambda+1} \operatorname{Ext}\left(Z\left(p^{\infty}\right), G / p^{\lambda} G\right)$ where $\lambda$ is a limit ordinal which is not cofinal with $\omega$. In [7], it was shown that the $S$-groups are the $p$-groups projective relative to a class of short exact sequences. Since the class of $S$-groups has a projective characterization and contains the totally projective $p$-groups, and since each totally projective $p$-group is $p^{\alpha}$-projective for some ordinal $\alpha$, it was conjectured that an $S$-group would also be $p^{\alpha}$-projective for some ordinal $\alpha$. However, it will be shown in this paper that an $S$-group is $p^{\alpha}$-projective only if it is totally projective; in fact, it is a summand of a group of the form Tor $A, B)$ where $A$ and $B$ are reduced $p$-groups only if it is totally projective, [Corollary 3.6]. These results will follow once it is shown that the invariant $k\left(p^{\lambda}\right.$, Tor $\left.(A, B)\right)$ is zero for all reduced $p$-groups $A$ and $B$, [Corollary 3.4]. Finally, it is shown that if $A, B$ and $\operatorname{Tor}(A, B)$ are three totally projective $p$-groups then either $A$ or $B$ is the direct sum of countable $p$-groups, [Corollary 3.8].

2. Notation. If $G$ is a group then let $c(G)$ denote the cotorsion hull of $G$, i.e., $c(G)=\operatorname{Ext}\left(Z\left(p^{\infty}\right), G\right)$ where $Z\left(p^{\infty}\right)$ is the divisible torsion p-group of $Q / Z$.

If $G$ is a reduced $p$-group then $l(G)$ will denote the length of $G$, i.e., $l(G)$ is the least ordinal for which $p^{l(G)} G=0$. Let $\Omega$ denote the first uncountable ordinal.

3. Results. Dr. R. Nunke has communicated orally to me the following result and proof which will be used in the proof of Lemma 3.2. 
THEOREM 3.1. Let $\left\{A_{\alpha}, \alpha \in \Gamma\right\}$ be a collection of p-groups, and $\lambda$ a limit ordinal such that $\lambda$ is not cofinal with $\omega$. If for each $\alpha \in \Gamma, p^{\lambda} c\left(A_{\alpha}\right)=0$, then $p^{\lambda} c\left(\oplus_{\alpha \in \Gamma} A_{\alpha}\right)=0$.

Proof. Let $A=\bigoplus_{\alpha \in \Gamma} A_{\alpha}$ and $e \in p^{\lambda} c(A)$ represent the exact sequence $e: 0 \rightarrow A \stackrel{\nu}{\rightarrow} M \rightarrow Z\left(p^{\infty}\right) \rightarrow 0$. For each $\alpha \in \Gamma$, the pushout sequence by the projection map $\pi_{\alpha}: A \rightarrow A_{\alpha}$ is $p^{\lambda}$-pure; consequently, there exists a map $\phi_{\alpha}: M \rightarrow A_{\alpha}$ such that the following diagram commutes.

$$
\begin{array}{rllllll}
e: 0 & \rightarrow & A & \rightarrow & M & \rightarrow & Z \\
& \pi_{\alpha} \downarrow \\
& A_{\alpha} & & & & &
\end{array}
$$

To show that the sequence $e$ splits, it must be shown that $\phi=\bigoplus_{\alpha \in \Gamma} \phi_{\alpha}$ : $M \rightarrow \oplus_{\alpha \in \Gamma} A_{\alpha}=A$ is a homomorphism, i.e., that for each $x$ in $M$ the set $\left\{\alpha \mid \phi_{\alpha}(x) \neq 0, \alpha \in \Gamma\right\}$ is finite. Suppose there exists an $x$ in $M$ and a sequence $\left\{\alpha_{t} \in \Gamma\right\}$ such that $\phi_{\alpha_{t}}(x) \neq 0$. Let $\beta$ be any ordinal which satisfies the inequalities $\lambda>\beta$ and $\beta>$ height of $\phi_{\alpha_{l}}(x)$ for each $i$. The ordinal $\beta$ exists since $\lambda>\alpha$, for each $i$ and $\lambda$ is a limit ordinal which is not cofinal with $\omega$. The sequence $e$ being $p^{\beta}$-pure and the group $Z\left(p^{\infty}\right)$ being divisible imply there exists an element $a$ in the subgroup $\nu(A)=$ $\nu\left(\oplus_{\alpha \in \Gamma} A_{\alpha}\right)$ of $M$, and an element $b$ in $p^{\beta} M$ for which $x=a+b,[3,87]$. For some $\alpha_{t}, \phi_{\alpha_{t}}(a)=0$; hence, $\phi_{\alpha_{t}}(x)=\phi_{\alpha_{t}}(b)$. This is a contradiction since the height of $\phi_{\alpha_{t}}(x)$ is less than $\beta$ whereas the height of $\phi_{\alpha_{t}}(b)$ is greater than or equal to $\beta$.

The following lemma will be used in the proof of Theorem 3.3.

Lemma 3.2. Let $\lambda$ be a limit ordinal which is not cofinal with $\omega$ and let $G$ be a p-group such that $p^{\lambda} G=0$. Then $p^{\lambda} c(\operatorname{Tor}(G, X))=0$ for any reduced group $X$.

Proof. There exists a reduced $p^{\lambda}$-injective group $I$ and a $p^{\lambda}$-pure sequence $0 \rightarrow G \rightarrow I \rightarrow U \rightarrow 0$, [3, 84]. It follows that $0 \rightarrow \operatorname{Tor}(G, X) \rightarrow$ $\operatorname{Tor}(I, X) \rightarrow \operatorname{Tor}(U, X) \rightarrow 0$ is a pure sequence of reduced groups, and the sequence

$$
0 \rightarrow c(\operatorname{Tor}(G, X)) \rightarrow c(\operatorname{Tor}(I, X)) \rightarrow c(\operatorname{Tor}(U, X)) \rightarrow 0
$$

is exact. Once it is shown that $p^{\lambda} c(\operatorname{Tor}(I, X))=0$, then the lemma is proved. To show this let $D$ be the injective hull of $X$ and $0 \rightarrow X \rightarrow D \rightarrow D^{\prime}$ $\rightarrow 0$ be the resulting exact sequence. Consequently, $0 \rightarrow \operatorname{Tor}(I, X) \rightarrow$ $\operatorname{Tor}(I, D) \rightarrow \operatorname{Tor}\left(I, D^{\prime}\right)$ is an exact sequence of reduced groups. The 
group $\operatorname{Tor}(I, D)$ is isomorphic to the direct sum of $\gamma$ copies of $t(I)$, the torsion subgroup of $I$, where $\gamma$ is the dimension of the $Z / p Z$-vector space $D[p]$, and $p^{\lambda} c(t(I)) \subseteq p^{\lambda} c(I)=0$. Hence, it follows from Theorem 3.1 that $p^{\lambda} c(\operatorname{Tor}(I, X)) \subseteq p^{\lambda} c(\operatorname{Tor}(I, D))=0$.

THEOREM 3.3. If $\lambda$ is a limit ordinal such that $\lambda$ is not cofinal with $\omega$, then $p^{\lambda} c(\operatorname{Tor}(A, B))=c\left(p^{\lambda} \operatorname{Tor}(A, B)\right)$ whenever $A$ and $B$ are reduced groups.

Proof. It need only be shown that $p^{\lambda} c(\operatorname{Tor}(A, B))$ is a subset of $c\left(p^{\lambda} \operatorname{Tor}(A, B)\right)$, since there is an exact sequence

$$
\begin{aligned}
0 & \rightarrow c\left(p^{\lambda} \operatorname{Tor}(A, B)\right) \rightarrow p^{\lambda} c(\operatorname{Tor}(A, B)) \\
& \rightarrow p^{\lambda} c\left(\operatorname{Tor}(A, B) / p^{\lambda} \operatorname{Tor}(A, B)\right) \rightarrow 0,
\end{aligned}
$$

$[1,56.1]$

The sequence

$$
0 \rightarrow \operatorname{Tor}\left(p^{\lambda} A, B\right) \rightarrow \operatorname{Tor}(A, B) \stackrel{\pi}{\rightarrow} \operatorname{Tor}\left(A / p^{\lambda} A, B\right) \stackrel{\delta}{\rightarrow}\left(p^{\lambda} A\right) \otimes B
$$

is exact. If $X$ is the image of $\pi$ and $Y$ the image of $\delta$, then $X$ and $Y$ are reduced subgroups of $\operatorname{Tor}\left(A / p^{\lambda} A, B\right)$ and $\left(p^{\lambda} A\right) \otimes B$, respectively. Therefore it follows that the sequences

$$
e: 0 \rightarrow c\left(\operatorname{Tor}\left(p^{\lambda} A, B\right)\right) \rightarrow c(\operatorname{Tor}(A, B)) \rightarrow c(X) \rightarrow 0
$$

and

$$
f: 0 \rightarrow c(X) \rightarrow c\left(\operatorname{Tor}\left(A / p^{\lambda} A, B\right)\right) \rightarrow c(Y) \rightarrow 0
$$

are exact sequences of reduced groups.

$$
p^{\lambda} c(\operatorname{Tor}(A, B)) \subseteq c\left(\operatorname{Tor}\left(p^{\lambda} A, B\right)\right)
$$

since

$$
p^{\lambda} c(X) \subseteq p^{\lambda} c\left(\operatorname{Tor}\left(A / p^{\lambda} A, B\right)\right)=0,
$$

[Lemma 3.2]. Similarly,

$$
p^{\lambda} c(\operatorname{Tor}(A, B)) \subseteq c\left(\operatorname{Tor}\left(A, p^{\lambda} B\right)\right) .
$$

The conclusion follows from the identity

$$
c\left(\operatorname{Tor}\left(A, p^{\lambda} B\right)\right) \cap c\left(\operatorname{Tor}\left(p^{\lambda} A, B\right)\right)=c\left(p^{\lambda} \operatorname{Tor}(A, B)\right),
$$

$[1,64.2]$. 
Corollary 3.4. If $G$ is a summand of $\operatorname{Tor}(A, B)$ where $A$ and $B$ are reduced p-groups, then $p^{\lambda} c(G)=c\left(p^{\lambda} G\right)$ (equivalently, $p^{\lambda} c\left(G / p^{\lambda} G\right)=0=$ $\left.k\left(p^{\lambda}, G\right)\right)$ for every limit ordinal $\lambda$ such that $\lambda$ is not cofinal with $\omega$. Consequently, if $G$ is $p^{\alpha}$-projective for some ordinal $\alpha$ then $p^{\lambda} c(G)=c\left(p^{\lambda} G\right)$ and $p^{\alpha} G=0$.

Proof. Since all the functors commute with direct sums, $p^{\lambda} c(G)=$ $c\left(p^{\lambda} G\right)$.

If $G$ is $p^{\alpha}$-projective for some ordinal $\alpha$ then there is a reduced group $H$ such that $p^{\alpha} H=0$ and $G$ is a summand of $\operatorname{Tor}(G, H)$. Hence, $p^{\lambda} c(G)$ $=c\left(p^{\lambda} G\right)$. Also, $p^{\alpha} G=0$ since $p^{\alpha} \operatorname{Tor}(G, H)=0$.

The equivalence of $p^{\lambda} c(G)=c\left(p^{\lambda} G\right)$ and $p^{\lambda} c\left(G / p^{\lambda} G\right)=0$ follows from the exact sequence $0 \rightarrow c\left(p^{\lambda} G\right) \rightarrow p^{\lambda} c(G) \rightarrow p^{\lambda} c\left(G / p^{\lambda} G\right) \rightarrow 0,[\mathbf{1}$, 56.1].

COROLlaRY 3.5. If $0 \rightarrow Z \rightarrow M \rightarrow H_{\lambda} \rightarrow 0$ is a sequence which represents $p^{\lambda}$ where $\lambda$ is a limit ordinal which is not cofinal with $\omega$, then the torsion subgroup of $M$ is not $p^{\alpha}$-projective for any ordinal $\alpha$.

Proof. Let $G$ be the torsion subgroup of $M$. $G$ is a $\lambda$-elementary $S$-group and in [6] it is shown that $k\left(p^{\lambda}, G\right) \neq 0$, [Corollary 3.4].

COROLlary 3.6. If $G$ is an $S$-group, then $G$ is $p^{\alpha}$-projective if and only if $G$ is a totally projective p-group and $p^{\alpha} G=0$. Also, $G$ is not a summand of a group $\operatorname{Tor}(A, B)$ where $A$ and $B$ are reduced groups, unless $G$ is totally projective.

Proof. This result follows from Corollary 3.4 and the fact that an $S$-group is totally projective if and only if $k\left(p^{\lambda}, G\right)=0$ for every limit ordinal $\lambda$ which is not cofinal with $\omega,[6]$.

THEOREM 3.7. If $A$ and $B$ are two totally projective p-groups such that $l(A) \geq l(B)=\alpha>\Omega$, then $\operatorname{Tor}(A, B)$ is not totally projective.

Proof. The proof will be by transfinite induction on the ordinal $\alpha$.

Case 1. $\alpha=\lambda+n+1$ where $\lambda$ is a limit ordinal and $n$ is a finite ordinal. Let $T$ be a $p^{\lambda+n}$-high subgroup of the group $A$ and $e: 0 \rightarrow T \rightarrow A$ $\rightarrow D \rightarrow 0$ be the resulting exact sequence. Since the sequence $e$ is $p^{\alpha}$-pure, 
[3, 92], and the group $\operatorname{Tor}(D, B)$ is $p^{\alpha}$-projective, $[3,82]$, the sequence $f$ : $0 \rightarrow \operatorname{Tor}(T, B) \rightarrow \operatorname{Tor}(A, B) \rightarrow \operatorname{Tor}(D, B) \rightarrow 0$ is $p^{\alpha}$-pure, [5, 2], and splits. Hence, $\operatorname{Tor}(A, B) \simeq \operatorname{Tor}(T, B) \oplus \operatorname{Tor}(D, B)$. The group $T$ is an $S$-group because $p^{\lambda} T$ and $T / p^{\lambda} T$ are both $S$-groups, [6,5.3]. Three subcases will now be considered. In each of the subcases $\operatorname{Tor}(A, B)$ will be shown to be not totally projective by showing that it has a summand which is not totally projective.

Case 1.1. $\alpha=\Omega+1$. The sequence $e$ being $p^{\alpha}$-pure implies that the sequence $0 \rightarrow \operatorname{Hom}\left(Z\left(p^{\infty}\right), D\right) \rightarrow p^{\Omega+1} c(T) \rightarrow p^{\Omega+1} c(A)=0$ is exact, [3, 89]. Since $D$ is a non-trivial divisible $p$-groups, $p^{\Omega} c(T) \neq c\left(p^{\Omega} T\right)=0$ and the group $T$ is not $p^{\Omega}$-projective, [Corollary 3.6]. Since $l(T)<l(B)$, $\operatorname{Tor}(T, B)$ is not totally projective, $[4,3.4]$.

Case 1.2. $\alpha>\Omega+1$ and the group $T$ is totally projective. Since $\Omega<l(T)=\lambda+n<l(B)$, induction is used to show that $\operatorname{Tor}(T, B)$ is not totally projective.

Case 1.3. $\alpha>\Omega+1$ and the group $T$ is not totally projective. By Corollary 3.6, the group $T$ is not $p^{\lambda+n}$-projective. Consequently, $\operatorname{Tor}(T, B)$ is not totally projective, $[4,3.4]$.

Case 2. $\alpha$ is a limit ordinal greater than $\Omega$. There exists a summand $W$ of $B$ such that the group $W$ is totally projective and $\Omega<l(W)<\alpha$, , 83.1(e)]. By induction, $\operatorname{Tor}(A, B)$ has a summand which is not a totally projective $p$-group.

Corollary 3.8. If $A, B$ and $\operatorname{Tor}(A, B)$ are three totally projective p-groups then either $A$ or $B$ is the direct sum of countable p-groups.

Proof. This corollary follows from Theorem 3.7 and noting that any totally projective $p$-group with length at most $\Omega$ is the direct sum of countable $p$-groups.

\section{REFERENCES}

[1] L. Fuchs, Infinite Abelian Groups, Vol. I, Academic Press, 1970.

[2] , Infinite Abelian Groups, Vol. II, Academic Press, 1973.

[3] P. A. Griffith, Infinite Abelian Group Theory, The University of Chicago Press, 1970.

[4] R. J. Nunke, On the Structure of Tor, in Proceedings of the Colloquium on Abelian Groups, Budapest, (1964), 115-124.

[5] , On the Structure of Tor II, Pacific J. Math., 22 (1967), 453-464. 
[6] R. B. Warfield, Jr., A Classification Theorem for Abelian p-groups, Trans. Amer. Math. Soc., 210 (1975), 149-168.

[7] B. D. Wick, A Projective Characterization for SKT-modules, Proc. Amer. Math. Soc., 80 (1980), 44-46.

Received November 9, 1981.

UNIVERSITY OF ALASKA

ANCHORAGE, AK 99508 


\title{
PACIFIC JOURNAL OF MATHEMATICS EDITORS
}

\author{
Donald BabBITT (Managing Editor) \\ University of California \\ Los Angeles, CA 90024 \\ Hugo Rossi \\ University of Utah \\ Salt Lake City, UT 84112 \\ C. C. Moore and Arthur Ogus \\ University of California \\ Berkeley, CA 94720
}

\author{
J. Dugundu \\ Department of Mathematics \\ University of Southern California \\ Los Angeles, CA 90089-1113
}

R. FINN and H. SAMELSON

Stanford University

Stanford, CA 94305

\section{ASSOCIATE EDITORS}
R. ARENS
E. F. BECKENBACH
B. H. NeumanN
F. WolF
K. YosHIDA (1906-1982)

\section{SUPPORTING INSTITUTIONS}

\author{
UNIVERSITY OF ARIZONA \\ UNIVERSITY OF BRITISH COLUMBIA \\ CALIFORNIA INSTITUTE OF TECHNOLOGY \\ UNIVERSITY OF CALIFORNIA \\ MONTANA STATE UNIVERSITY \\ UNIVERSITY OF NEVADA, RENO \\ NEW MEXICO STATE UNIVERSITY \\ OREGON STATE UNIVERSITY
}

\author{
UNIVERSITY OF OREGON \\ UNIVERSITY OF SOUTHERN CALIFORNIA \\ STANFORD UNIVERSITY \\ UNIVERSITY OF HAWAII \\ UNIVERSITY OF TOKYO \\ UNIVERSITY OF UTAH \\ WASHINGTON STATE UNIVERSITY \\ UNIVERSITY OF WASHINGTON
}

The Supporting Institutions listed above contribute to the cost of publication of this Journal, but they are not owners or publishers and have no responsibility for its content or policies.

Mathematical papers intended for publication in the Pacific Journal of Mathematics should be in typed form or offset-reproduced (not dittoed), double spaced with large margins. Please do not use built up fractions in the text of the manuscript. However, you may use them in the displayed equations. Underline Greek letters in red, German in green, and script in blue. The first paragraph must be capable of being used separately as a synopsis of the entire paper. In particular it should contain no bibliographic references. Please propose a heading for the odd numbered pages of less than 35 characters. Manuscripts, in triplicate, may be sent to any one of the editors. Please classify according to the scheme of Math. Reviews, Index to Vol. 39. Supply name and address of author to whom proofs should be sent. All other communications should be addressed to the managing editor, or Elaine Barth, University of California, Los Angeles, California 90024.

There are page-charges associated with articles appearing in the Pacific Journal of Mathematics. These charges are expected to be paid by the author's University, Government Agency or Company. If the author or authors do not have access to such Institutional support these charges are waived. Single authors will receive 50 free reprints; joint authors will receive a total of 100 free reprints. Additional copies may be obtained at cost in multiples of 50 .

The Pacific Journal of Mathematics is issued monthly as of January 1966. Regular subscription rate: $\$ 132.00$ a year (6 Vol., 12 issues). Special rate: $\$ 66.00$ a year to individual members of supporting institutions.

Subscriptions, orders for numbers issued in the last three calendar years, and changes of address should be sent to Pacific Journal of Mathematics, P.O. Box 969, Carmel Valley, CA 93924, U.S.A. Old back numbers obtainable from Kraus Periodicals Co., Route 100, Millwood, NY 10546.

The Pacific Journal of Mathematics ISSN 0030-8730 is published monthly by the Pacific Journal of Mathematics at P.O. Box 969, Carmel Valley, CA 93924. Application to mail at Second-class postage rates is pending at Carmel Valley, California, and additional mailing offices. Postmaster: Send address changes to Pacific Journal of Mathematics, P. O. Box 969, Carmel Valley, CA 93924.

PUBLISHED BY PACIFIC JOURNAL OF MATHEMATICS. A NON-PROFIT CORPORATION

Copyright $\odot 1984$ by Pacific Journal of Mathematics 


\section{Pacific Journal of Mathematics}

\section{Vol. 112, No. $2 \quad$ February, 1984}

Kenneth F. Andersen and Wo-Sang Young, On the reverse weak type inequality for the Hardy maximal function and the weighted classes

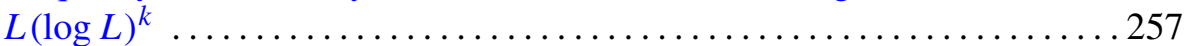

Richard Eugene Bedient, Double branched covers and pretzel knots ..... 265

Harold Philip Boas, Holomorphic reproducing kernels in Reinhardt

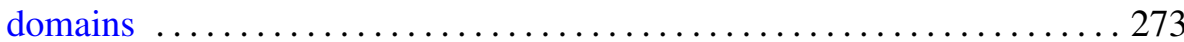

Janey Antonio Daccach and Arthur Gabriel Wasserman, Stiefel's theorem and toral actions ................................. 293

Michael Fried, The nonregular analogue of Tchebotarev's theorem ....... 303

Stanley Joseph Gurak, Minimal polynomials for circular numbers . . . . . . 313

Norimichi Hirano and Wataru Takahashi, Nonlinear ergodic theorems for an amenable semigroup of nonexpansive mappings in a Banach space . . 333

Jim Hoste, Sewn-up $r$-link exteriors . . . . . . . . . . . . . . . . . . . 347

Mohammad Ahmad Khan, The existence of totally dense subgroups in

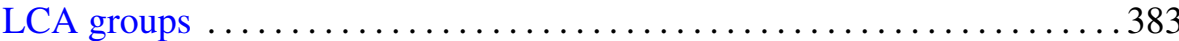

Mieczysław Kula, Murray Angus Marshall and Andrzej Sładek, Direct

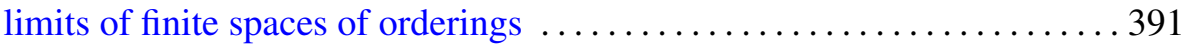

Luis Montejano Peimbert, Flat Hilbert cube manifold pairs ........... 407

Steven C. Pinault, An a priori estimate in the calculus of variations . . . . . 427

McKenzie Y. K. Wang, Some remarks on the calculation of Stiefel-Whitney classes and a paper of Wu-Yi Hsiang's

Brian Donald Wick, The calculation of an invariant for Tor . . 445

Wolfgang Wollny, Contributions to Hilbert's eighteenth problem 